\title{
Spatially-resolved STEM-EELS of waveguide modes
}

\author{
David Kordahl ${ }^{1}$, Duncan Alexander ${ }^{2}$ and Christian Dwyer ${ }^{3}$ \\ ${ }^{1}$ Centenary College of Louisiana, Shreveport, Louisiana, United States, ${ }^{2}$ Ecole Polytechnique Federale de Lausanne, \\ United States, ${ }^{3}$ Electron Imaging and Spectroscopy Tools, United States
}

The innovations in STEM-EELS instrumentation in recent years have been truly impressive, enabling significant advances in the ability to probe nanomaterials, and even giving rise to the new field of vibrational STEM-EELS [1,2]. Notwithstanding these advances, it remains that the vast majority of works concern collective or single-particle excitations that are well-described by the electrostatic approximation. In this approximation, which can be formally obtained as the limit of Maxwell's equations in which the speed of light tends to infinity, the effects of retardation and magnetic fields are neglected.

Here, by contrast, we explore cases in STEM-EELS mapping in which the electrostatic approximation is fundamentally inadequate. When the beam energy is above the Cherenkov threshold, and the geometric dimensions of the nanomaterial sample are comparable to the wavelength of light in the material, low-loss $(\lesssim 5 \mathrm{eV})$ STEM-EELS maps from guided light modes may be observed. We demonstrate such observations for amorphous silicon discs and offer a relatively simple interpretation of the results based on the waveguide modes of a cylinder.

Alongside the above-mentioned advances in instrumentation, there have also been steady advances in the theory and computation of STEM-EELS plasmon mapping, e.g., using the discrete dipole approximation or the boundary element method. While these implementations certainly do allow fully electrodynamic simulations (i.e., based on the full set of Maxwell's equations including retardation and magnetic fields), it is striking how successful the electrostatic approximation is in capturing the essential physics in most cases. Indeed, apart from nanomaterials like photonic crystals or metallic arrays that are specifically tailored to generate electrodynamic effects, comparisons of the electrostatic and electrodynamic approaches [3,4,5] have reported only modest differences, with electrodynamic effects often just redshifting modal frequencies and/or decreasing the intensities of spectral features by only a few percent.

By contrast, recent STEM-EELS measurements on 100-500 nm diameter amorphous silicon discs [6,7] show electrodynamic effects that cannot be captured by the electrostatic approximation. While the low-loss $(<2.4 \mathrm{eV})$ STEMEELS maps in question superficially resemble the surface modes commonly observed in dielectric nanomaterials, surface modes in the electrostatic approximation require the real part of the dielectric function to be negative, whereas silicon has a dielectric function that is positive and increasing in the energy range of interest. Rather, the STEM-EELS maps in question are attributable to guided light modes, which demand a fully electrodynamic description.

We first illustrate the point in Fig. 1, where we show simulated EEL spectra for silicon "ribbons" which are $100 \mathrm{~nm}$ thick (beam direction), have widths between 10 and $1000 \mathrm{~nm}$, and are infinite in the other lateral direction. Results are shown for beam energies of 10, 30 or $300 \mathrm{keV}$. Electrodynamic corrections become increasingly important with increasing beam energy and increasing ribbon width. The sharp peaks in the electrodynamic prediction indicate the presence of TE modes and the corresponding STEM-EELS maps (not shown) exhibit distinct standing-wave patterns. Note that electrodynamic corrections are most significant in the lower energy range where $\operatorname{Re}[\varepsilon]>0$, whereas in the higher energy range where $\operatorname{Re}[\varepsilon]<0$, the electrostatic and electrodynamic models match qualitatively.

Fig. 2 shows experimental and simulated STEM-EELS maps for a $300 \mathrm{~nm}$ diameter silicon disc. The simulations are based on the waveguide modes of an infinitely long cylinder (viewed along its axis). These modes are labelled in Fig. 2. Despite the idealization of the experimental geometry, many of the features in the experimental maps are reproduced by the cylinder waveguide model. The lowest-energy modes in each disc resemble the HE11 waveguide modes, and the next-to-lowest-energy modes resemble the TM01 waveguide modes.

As will be discussed in detail, such comparisons enable us to establish guidelines for predicting when a STEM-EELS signal is fundamentally electrodynamic (for which any electrostatic interpretation will fail), versus those for which an electrostatic interpretation should work well [8]. 

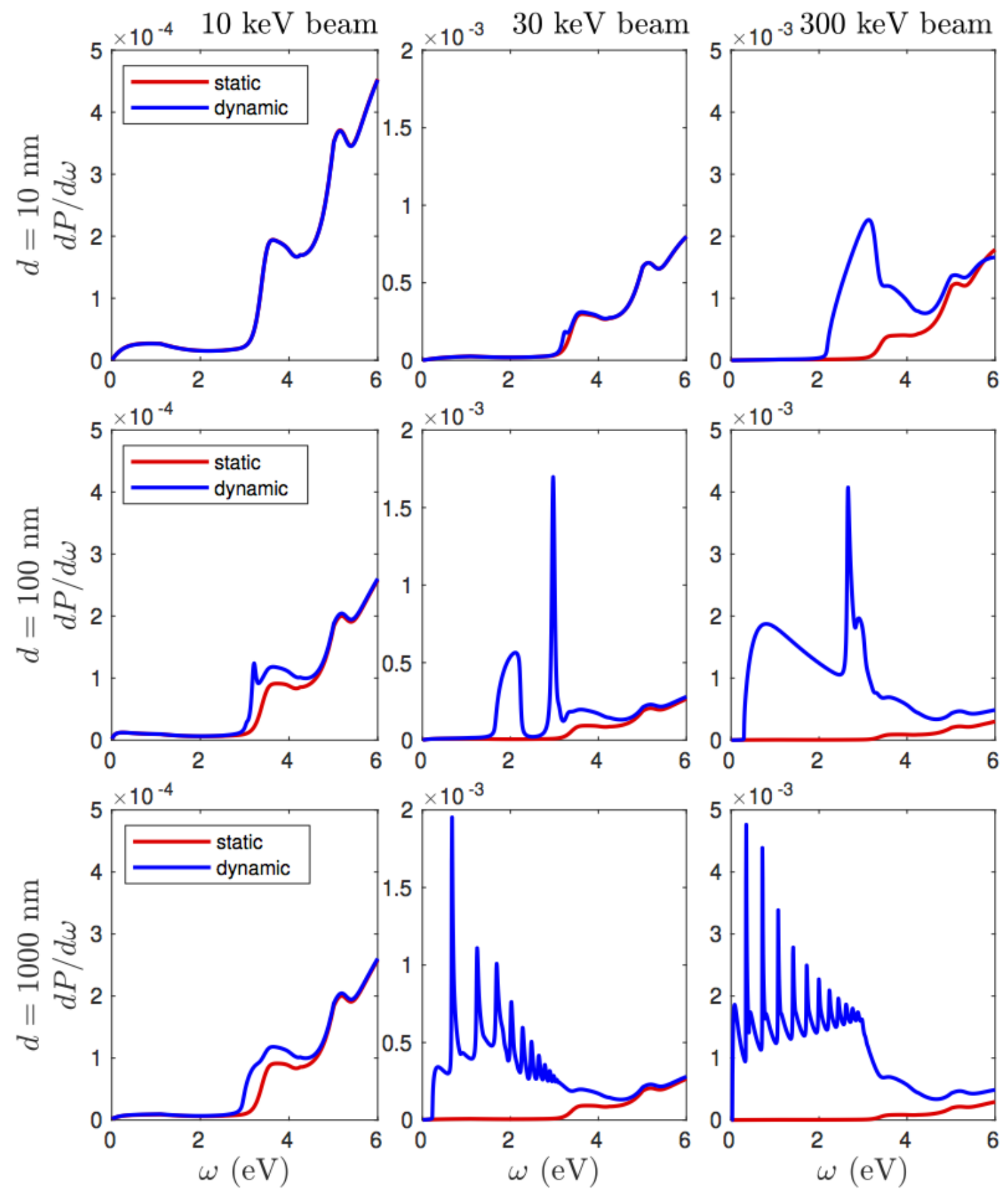

Figure 1. Comparison of EEL spectra simulated using electrostatic and electrodynamic approaches (plotted in red and blue, respectively), for silicon "ribbons" of varying widths d (indicated), and various beam energies (indicated). 

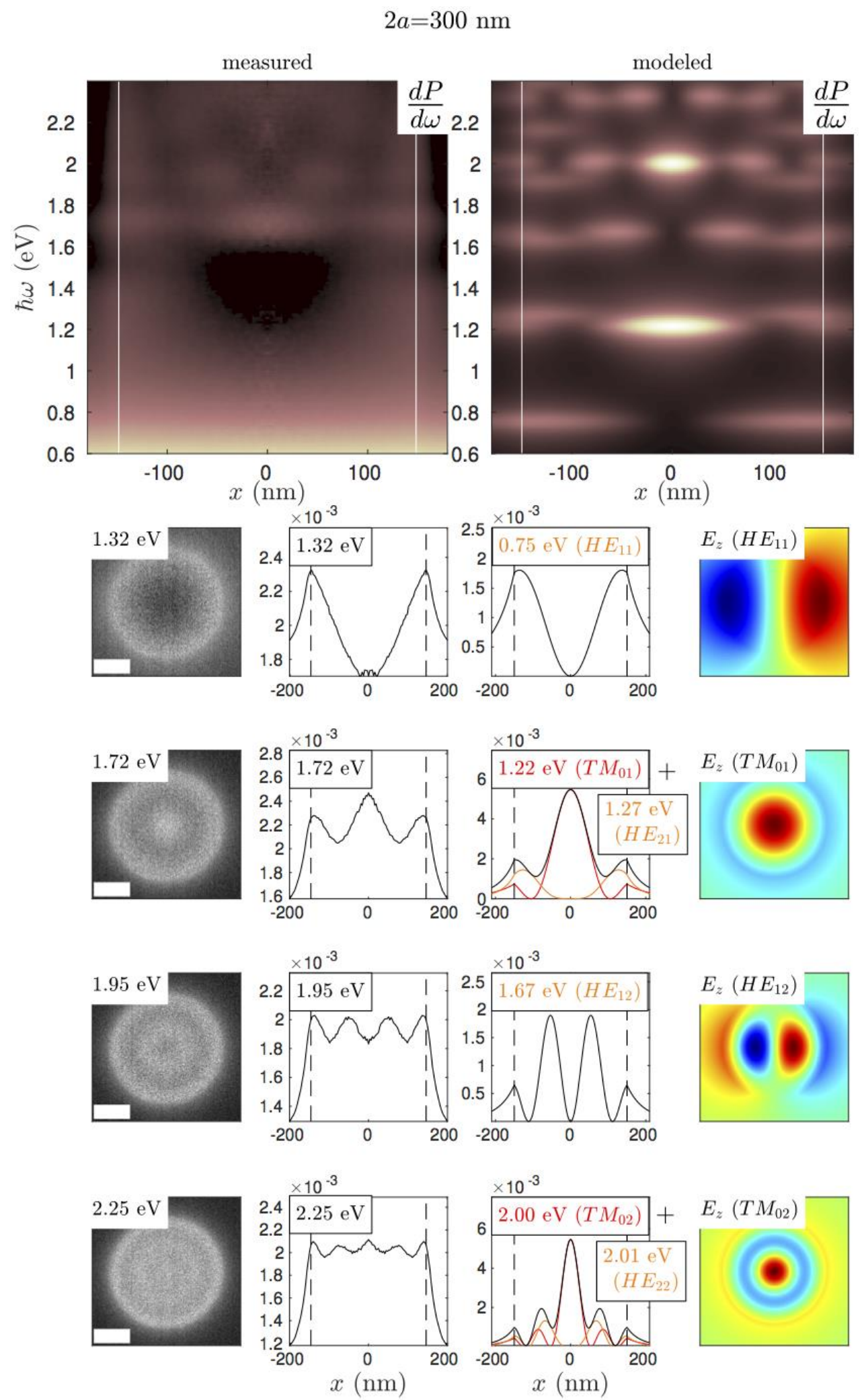

Figure 2. Experimental (left column) and simulated (right column) STEM-EELS maps of a $300 \mathrm{~nm}$ silicon disc. Top row shows 2D spatial-energy plots (from radially averaged data). Far left: experimental spectral maps using $0.1 \mathrm{eV}$ window. Center left: experimental linescans using reflected radial averages. Center right: radial linescan from simulation. Far right: In-plane map of Ez from simulation. Dashed lines represent cylinder edges. Scale bars represent $100 \mathrm{~nm}$. 


\section{References}

[1] O. L. Krivanek et al., Nature 514, 209 (2014).

[2] T. Miyata et al., Microscopy 63, 377 (2014).

[3] A. A. Govyadinov et al., Nature Communications 8 (2017).

[4] F. J. García de Abajo, Physical Review. B 59, 3095 (1999).

[5] A. Losquin and M. Kociak, ACS photonics 2, 1619 (2015).

[6] V. Flauraud and D. T. Alexander, Microscopy and Microanalysis 25, 634 (2019).

[7] V. Flauraud, F. Demming-Janssen, and D. Alexander, (2021), in preparation.

[8] D. Kordahl, D.T.L. Alexander, C. Dwyer, (2021), submitted. 